\title{
The Association between Regional Fat Distribution and Acute Mountain Sickness in Young Hikers
}

(이(1) (

Authors

Devon A. Dobrosielski, Michelle Guadagno, Phillip Phan

Affiliation

Kinesiology, Towson University, Towson, United States

\section{Key words}

dual-energy X-ray absorptiometry, Lake Louise score, high-altitude recreation, abdominal visceral fat, central adiposity, hiking

received 23.10 .2016

revised 22.12 .2016

accepted 24.12.2016

Bibliography

DOI http://dx.doi.org/10.1055/s-0043-101371

Sports Medicine International Open 2017; 2: E74-E79

(c) Georg Thieme Verlag KG Stuttgart · New York

ISSN 2367-1890

\section{Correspondence}

Dr. Devon Alexander Dobrosielski, PhD

Kinesiology

Towson University

8000 York Road

21204, Towson

United States

Tel.: + 1/410/704 5604, Fax: +1/410/7043912

ddobrosielski@towson.edu

\begin{abstract}
Acute mountain sickness (AMS) can occur upon rapid ascent from low to high altitude. This study examined the association between central adiposity and the development of AMS in young adults during a high-altitude hike. Total and regional body fat were measured at sea level using dual-energy $\mathrm{X}$-ray absorptiometry. Within $24 \mathrm{~h}$ of arriving in Cusco, Peru (3 400 meters) participants embarked on a 14-mile hike across the Andes Mountain range in southern Peru. Symptoms of AMS were assessed using the Lake Louise score at $24 \mathrm{~h}$ ( 3400 meters), $29 \mathrm{~h}$ (4 100 meters), $34 \mathrm{~h}$ ( 3800 meters) and $53 \mathrm{~h}$ ( 2900 meters). 14 participants (mean age $21 \pm 2$ years; women: 11 , men: 3 ) completed the study. The number of participants exhibiting at least mild AMS increased from $6(54 \%)$ at 3400 meters to $9(64 \%)$ at 3800 meters. A higher AMS score at 4100 meters was associated with greater android $(r=0.72, p<0.01)$, trunk $(r=0.73, p<0.01)$ and total body $(r=0.71, p<0.01)$ fat, but not with total body fat $\%(r=0.39, p=0.16)$. Our findings suggest that central obesity, but not total body fat per se, may be an important factor in the development of AMS.
\end{abstract}

\section{Introduction}

The number of international travelers to cities located in the South American Andes Mountains has increased substantially over the past decade. In 2008, Cusco Peru (elevation 3400 meters) was the destination of one million foreign visitors [1]. It is estimated that approximately $75 \%$ of these travelers ascend to Cusco in flights departing from sea level and lasting one hour [1]. This rapid ascent from low to high altitude (>2500 meters) often results in acute mountain sickness (AMS), a syndrome characterized by a headache and the presence of nausea, lassitude, shortness of breath, dizziness or difficulty sleeping.

Since prevention of AMS is preferable to treatment, much attention has focused on identifying potential risk factors of this syndrome [2-4]. It is generally understood that AMS is precipitated by a deficiency in the acclimatization to altitude-induced hypoxia and its occurrence is positively related to speed of ascent and altitude attained [5]. In addition, there is some evidence that obesity is associated with the development of AMS [2, 6-9], although this finding has not been consistent across all studies [3, 4, 10, 11]. For ex- ample, Wu et al. [2] examined the incidence of AMS among 11000 workers at altitudes between 3500 meters and 5000 meters during the construction of the Qinghai-Tibet railroad. They found that overweight workers (BMI $\geq 25 \mathrm{~kg} / \mathrm{m}^{2}$ ) were 3 times more likely to suffer from AMS than normal weight workers. In a regression analysis, obesity was a significant predictor of AMS, independent of rate of ascent, heavy physical exercise, age, absolute altitude reached and magnitude of oxygen desaturation. More recently, Yang et al. [8] assessed AMS in 262 men (120 obese and 142 non-obese) 12 and $24 \mathrm{~h}$ after rapidly ascending by air to an altitude of $3658 \mathrm{me}-$ ters. AMS scores among obese men (BMI $\left.>28 \mathrm{~kg} / \mathrm{m}^{2}\right)$ were higher than non-obese men (BMI between 18.5 and $23.9 \mathrm{~kg} / \mathrm{m}^{2}$ ) at both time points. In contrast, Schneider et al. [3] administered the Environmental Symptom Questionnaire (ESQ) to mountaineers who had ascended to an altitude of 4559 meters in the Alps and found no difference in AMS prevalence between those subjects with a BMI equal to or greater than 26 and those with a BMI below 26. Similarly, Wagner et al.[10] reported that $33 \%$ of Mt. Whitney (elevation 4419 meters) summiteers met the criteria for AMS, but a logistic 
regression model revealed that age, not BMI, was the only physical characteristic that predicted AMS occurrence.

We note that all of these previous experiments used BMI as a surrogate for "body fatness". Ri-Li and colleagues [9] were the first to prospectively evaluate the association between obesity, assessed by hydrostatic weighing, and the development of AMS. 9 obese (total body fat $\geq 30 \%$ ) and 10 non-obese (total body fat $<25 \%$ ) men spent $24 \mathrm{~h}$ in a hypobaric environmental chamber, in which the barometric pressure was held at $483 \mathrm{~mm} \mathrm{Hg}$ (a pressure equivalent to an altitude of 3658 meters). The average AMS scores among the obese men increased more rapidly with time spent in the chamber compared to the non-obese men. Moreover, nighttime arterial oxygen saturation $\left(\mathrm{SaO}_{2}\right)$ levels were lower in the obese men, which suggests these men experienced sleep-disordered breathing [12]. Because central obesity is the strongest predictor of sleep-disordered breathing [13], we hypothesize that individuals who distribute fat centrally are more susceptible to AMS. Yet, the aforementioned studies lacked the methodological approach needed to examine this hypothesis. Accordingly, the purpose of the present study was to examine the association between total and regional body fat, assessed with dual-energy X-ray absorptiometry (DXA), and the development of AMS in a group of young adults trekking across the Andes Mountains region of southern Peru.

\section{Methods}

\section{Participants}

University students who were enrolled in a study-abroad program to Peru volunteered for this study. All participants were free of cardiovascular or pulmonary diseases and were non-smokers. No participants reported taking any medications to aid altitude acclimatization (e. g., sildenafil, acetazolamide) prior to or during the trip. Participants were sea level natives with no history of traveling to altitudes above 2000 meters. The authors have read and understand the ethical standards document of the journal and thereby assure that the present study was conducted in accordance with recognized ethical standards and national/international laws [14]. Each participant provided written informed consent. The Towson University Institutional Review Board approved the study.

\section{Experimental design}

The study was conducted during June of 2014. At sea level (Towson, MD, USA; elevation 141 meters), body composition was determined by DXA and aerobic capacity was assessed during an exercise test using expired gas analysis. Within one week of completing these measurements, participants traveled to Lima, Peru (sea level) and then flew to Cusco, Peru (elevation, 3400 meters). $24 \mathrm{~h}$ after arriving in Cusco, participants embarked on a 14-mile hike across the Andes Mountain range in southern Peru. The hike began in Tambomachay (elevation, 3750 meters) and reached an altitude of 4100 meters in $5 \mathrm{~h}$. An additional $2 \mathrm{~h}$ was spent climbing to 4300 meters, after which participants descended to Paucarcancha (elevation, 3800 meters) and set up a campsite. Following an overnight stay, the participants descended the mountain for approximately $5 \mathrm{~h}$ until they reached the town of Lamay (elevation, 2900 meters). $\mathrm{SaO}_{2}$ was measured by pulse oximetry (Vive Precision) and symptoms of AMS were assessed according to guidelines established by the Lake Louise AMS consensus report at $24 \mathrm{~h}$ (3400 meters), $29 \mathrm{~h}$ (4 100 meters), $34 \mathrm{~h}$ (3800 meters) and $53 \mathrm{~h}$ (2900 meters) after arrival in Cusco [15].

\section{Body composition assessment}

Height and weight were measured in metric units using a stadiometer and digital scale, respectively. Total body DXA scans were acquired by the same operator in the frontal plane using the Lunar iDXA in the standard mode (GE Healthcare, Madison, WI, USA). The DXA scanner underwent a daily quality assurance procedure according to manufacture guidelines. The precision error for total body analysis is $0.010 \mathrm{~g} / \mathrm{cm}^{2}$. Bone landmarks were used to define body regions (e. g., arms, legs, trunk). Abdominal visceral fat was computed over the android region and reported as grams. The android region is roughly $10 \mathrm{~cm}$ in height, extending from the iliac crest toward the head, a height that is $20 \%$ of the distance from the iliac crest to the base of the mandible. Scans were analyzed with the enCORE software (version 14.0).

\section{Assessment of aerobic capacity}

Peak oxygen consumption was determined with a Bruce treadmill protocol [16] using a TrueOne 2400 Metabolic Measurement System (Parvo Medics, Sandy, UT, USA) with breath-by-breath analysis. Maximal heart rate was defined as the highest value recorded during the test and $\mathrm{VO}_{2 \max }$ was represented by the highest observable $\mathrm{VO}_{2}$ averaged over $60 \mathrm{~s}$. To ensure that each participant achieved maximal exertion during the treadmill test, the following 3 criteria were met by every participant: (a) maximal achieved heart rate of at least $85 \%$ age predicted maximum; (b) a respiratory exchange ratio (RER) of at least 1.1 ; and $(c)$ a respiratory rate $>35$ breaths per minute [17].

\section{Assessment of AMS}

All participants completed an AMS self-report questionnaire. The items in the questionnaire include symptoms of headache, gastrointestinal symptoms, fatigue/weakness, dizziness or lightheadedness, and difficulty sleeping. Each of these symptoms was rated on a scale of 0 to 3 , with 0 representing no symptoms; 1 , mild symptoms; 2 , moderate symptoms; and 3 , severe symptoms. A diagnosis of AMS was based on the presence of a headache plus at least one other symptom and an overall score of 3-5 (defined as mild AMS) or 6-15 (defined as severe AMS). The sensitivity and specificity of the questionnaire has been reported elsewhere [18].

\section{Overnight sleep monitoring}

3 Morpheus Ox (WideMed Ltd, Herzliya, Israel) sleep monitoring devices were available to obtain polysomnography (PPG) derived signals during sleep as described elsewhere [19] Briefly, PPG uses a pulse oximeter to detect blood volume changes in the microvascular bed of the fingertip, and proprietary software $[20,21]$ is used to analyze the PPG for baseline variations, envelope and rate. These parameters are combined to generate a PPG-derived respiration (PDR) waveform that is correlated with saturation reductions to detect a clinically relevant apnea-hypopnea index (AHI). In this study we report a $4 \%$ (2007 AASM criteria) oxygen desaturation index (ODI4) for defining hypopneas. Furthermore, the PPG software 
- Table 1 Participant demographics.

\begin{tabular}{|c|c|c|c|}
\hline Variable & $\begin{array}{l}\text { Male } \\
(n=3)\end{array}$ & $\begin{array}{l}\text { Female } \\
(n=11)\end{array}$ & P-value \\
\hline \multicolumn{4}{|l|}{ Race or ethnicity, $n$} \\
\hline Asian & 1 & 2 & \\
\hline Black & 0 & 1 & \\
\hline White & 2 & 8 & \\
\hline Age (yrs) & $19.3 \pm 1.5$ & $21.2 \pm 1.7$ & 0.12 \\
\hline Weight (kg) & $71.0 \pm 17.6$ & $66.3 \pm 13.2$ & 0.5 \\
\hline Height (cm) & $170.6 \pm 3.0$ & $165.1 \pm 6.0$ & 0.15 \\
\hline BMI $(\mathrm{kg} / \mathrm{m} 2)$ & $24.2 \pm 5.1$ & $23.7 \pm 4.5$ & 0.86 \\
\hline Total body fat (\%) & $21.0 \pm 4.0$ & $32.0 \pm 5.0$ & 0.01 \\
\hline Total body fat $(\mathrm{kg})$ & $15.9 \pm 9.4$ & $20.9 \pm 7.5$ & 0.35 \\
\hline $\begin{array}{l}\text { Abdominal visceral } \\
\text { fat }(\mathrm{g})\end{array}$ & $213 \pm 211$ & $117 \pm 163$ & 0.41 \\
\hline Trunk fat $(\mathrm{g})$ & $7621 \pm 5438$ & $9612 \pm 4348$ & 0.51 \\
\hline $\begin{array}{l}\text { Maximal } \mathrm{VO}_{2} \\
(\mathrm{ml} / \mathrm{kg} / \mathrm{min})\end{array}$ & $50.8 \pm 4.2$ & $38.7 \pm 6.3$ & $<0.01$ \\
\hline $\begin{array}{l}\text { RER at max exercise } \\
\left(\mathrm{VCO}_{2} / \mathrm{VO}_{2}\right)\end{array}$ & $1.2 \pm 0.9$ & $1.2 \pm 0.9$ & 0.52 \\
\hline $\begin{array}{l}\text { RR at max exercise } \\
\text { (breaths per min) }\end{array}$ & $53 \pm 5$ & $52 \pm 6$ & 0.83 \\
\hline $\begin{array}{l}\text { HR at max exercise } \\
\text { (beats per min) }\end{array}$ & $192 \pm 8$ & $191 \pm 9$ & 0.89 \\
\hline
\end{tabular}

platform facilitates the detection and measurement of respiratory events, sleep/wake epochs, and total sleep time [20, 21]. Respiratory events occurring during sleep epochs are averaged over total sleep time to generate the $\mathrm{AHI}$ value. The women in the sample with the highest, lowest and median total body fat values were assigned to wear the device at sea level, and at 3400 meters.

\section{Statistical analysis}

Primary outcomes were inspected for normality using stem and leaf plots and the Shapiro-Wilk test. All data are presented as median (interquartile range) unless specified otherwise. Comparisons of body fat parameters according to AMS score were made using the Wilcoxon-Mann-Whitney test (non-parametric) or independent samples t-test (normally distributed data). The relation between AMS scores at each altitude with non-normally distributed body composition parameters were examined using Spearman correlations (rho). Pearson product-moment correlations were used to compare AMS scores with body composition parameters that were normally distributed. The level of statistical significance was set at $\mathrm{p}<0.05$ (2-tailed). All analyses were conducted using STATA, version 12 (StataCorp LP, College Station, TX, USA).

\section{Results}

14 participants ( 3 men, 11 women, age $21 \pm 2$ years) completed all aspects of the study. $\triangleright$ Table 1 displays the participant characteristics according to sex. Of note, women had higher total percent body fat compared with men $(p=0.01)$ and achieved a lower peak oxygen consumption during the exercise test $(p<0.01)$. No differ-

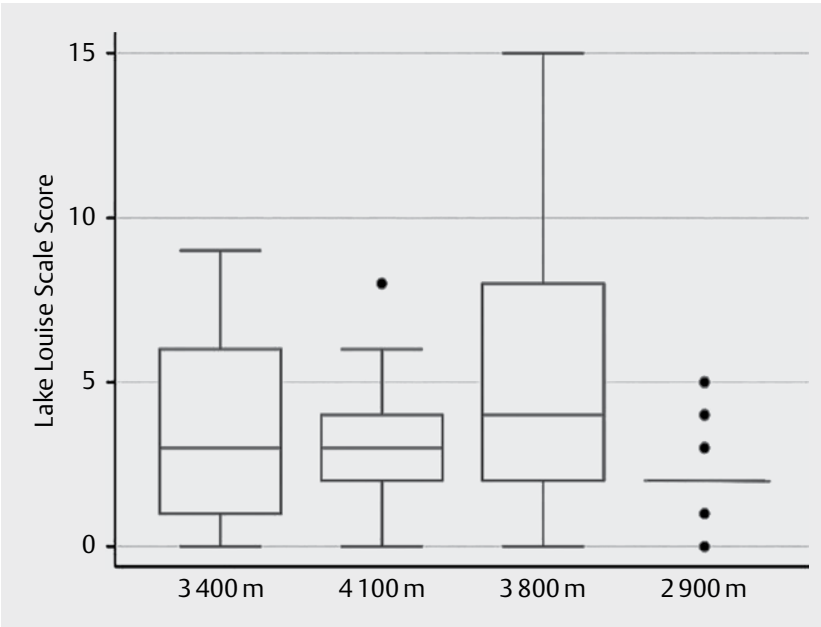

- Fig. 1 Distribution of AMS scores at each altitude with the group.

ences in abdominal visceral and trunk fat between men and women were observed.

Results of the Shapiro-Wilk test demonstrate that apart from abdominal visceral fat $(p<0.01)$, all data were normally distributed. At 3400 meters and 4100 meters, 8 participants (57\%) reported at least mild AMS (AMS score $\geq 3$ ). The incidence increased to 10 participants (71\%) at an altitude of 3800 meters. At 2900 meters, only 3 individuals ( $21 \%$ ) reported an AMS score $\geq 3$. The mean $\mathrm{SaO}_{2}$ of the sample at 3400 meters, 4,100 meters, 3,800 meters and 2,900 meters was $88.9 \pm 6.0,82.5 \pm 5.4,82.4 \pm 5.1$ and $94.1 \pm 1.8 \%$, respectively. - Fig. 1 displays the distribution of AMS scores across the sample using boxplots. The bottom and top of each box represent the lower $25^{\text {th }}$ quartile of scores and the $75^{\text {th }}$ quartile of scores, respectively. The middle line represents the median score.

Body composition parameters from DXA are displayed in - Table 2 according to self-reported AMS at 3800 meters. No differences in total body fat or \% total body fat were noted between those with and without severe AMS. No differences in the amount of android fat $[1919 \mathrm{~g}(975-2872 \mathrm{~g})$ vs. $1031 \mathrm{~g}(635-1409 \mathrm{~g})$, $\mathrm{p}=0.056]$ trunk fat $[11778 \mathrm{~g}(7890-1724 \mathrm{~g})$ vs. 8401 (4978-9 982 g), $\mathrm{p}=0.069$ ] and abdominal visceral fat [295 g (89$516 \mathrm{~g})$ vs. $55 \mathrm{~g}(24-113 \mathrm{~g}), \mathrm{p}=0.089)$ ] were noted between those who reported severe AMS compared to those without. However, individuals who reported an AMS score of 6 or greater at 3800 meters had a higher BMI compared to those who reported no or mild AMS at 3800 meters [27.5 (23.9-32.4) vs. 22.3 (20.0-23.0), $\mathrm{p}=0.012]$.

Associations between markers of body fat parameters, maximal oxygen consumption and AMS scores at various altitudes are displayed in $\triangleright$ Table 3 . As expected, total body fat was highly correlated with trunk $(r=0.99, p<0.01)$, android fat $(r=0.95, p<0.01)$ and maximal oxygen consumption $(r=-0.75, p<0.01)$. A spearman correlation revealed a significant relationship with abdominal visceral fat and self-reported AMS score at 3800 meters ( $r h o=0.58, p=0.03$ ). A higher AMS score at 4100 meters was associated with greater android $(r=0.72, p<0.01)$, trunk $(r=0.73, p<0.01)$ and total body $(r=0.71, p<0.01)$ fat, but not with total percent body fat $(r=0.39$, 
- Table 2 Body composition comparisons between participants with and without severe AMS at $3800 \mathrm{~m}$.

\begin{tabular}{|l|c|c|c|}
\hline Variable & $\begin{array}{c}\text { AMS<6 } \\
(\mathbf{n = 1 0})\end{array}$ & $\begin{array}{c}\text { AMS } \geq \mathbf{6} \\
(\mathbf{n = 4})\end{array}$ & P-value \\
\hline Android fat $(\mathrm{g})$ & $1031(635-1409)$ & $1919(975-2872)$ & 0.056 \\
\hline Trunk fat $(\mathrm{g})$ & $8401(4978-9982)$ & $22806(16745-33109)$ & 0.069 \\
\hline Total fat $(\mathrm{g})$ & $19254(13104-21422)$ & $295(89-516)$ & 0.129 \\
\hline Visceral fat $(\mathrm{g})$ & $55(24-113)$ & $29(25-36)$ & 0.089 \\
\hline Body fat \% & $33(27-34)$ & $27.5(23.9-32.4)$ & 0.887 \\
\hline BMl $\left(\mathrm{kg} / \mathrm{m}^{2}\right)$ & $22.3(20.0-23.0)$ & & 0.012 \\
\hline \multicolumn{2}{|l}{} \\
\hline
\end{tabular}

$p=0.16)$ or maximal oxygen consumption $(r=-0.24, p=0.40)$. A significant association was observed between $\mathrm{BMl}$ and self-reported AMS score at 4100 meters $(r=0.77, p<0.01)$, but not at 3400 meters $(r=0.47, p=0.09)$ or $3800(r=0.50, p=0.07)$.

Total body fat percentages and maximal aerobic capacities for the 3 women who underwent PPG monitoring were 23,34 and $43 \%$ and $39.4 \mathrm{ml} / \mathrm{kg} / \mathrm{min}, 46.5 \mathrm{ml} / \mathrm{kg} / \mathrm{min}$ and $27.6 \mathrm{ml} / \mathrm{kg} / \mathrm{min}$, respectively. The mean overnight $\mathrm{SaO}_{2}$ at sea level among these women was $95.7 \%$. None of the women experienced sleep-disordered breathing at sea level, defined as an ODI $>5$ event/hour of sleep. Importantly, at 3400 meters, the leanest woman experienced an ODI of 12 events/hour, while the woman with the highest \% body fat exhibited an ODI of 52 events/hour. Data at 3400 meters for the third woman was excluded from analysis because the PPG estimate sleep time was $<2 \mathrm{~h}$.

\section{Discussion}

Among a group of young adults hiking in the Andes mountain range, we observed a $57 \%$ incidence rate of AMS at 3400 meters that increased to $71 \%$ at 3800 meters. There was a trend for those individuals who reported severe AMS to have greater central adiposity compared to those who reported no or mild AMS. Further, we found greater amounts of regional and total body fat were associated with increased AMS severity.

While there are several studies that have not found BMI to be a significant predictor of AMS among trekkers to very high altitudes (between 4419 and 5671 meters) $[3,4,10,11]$, our data corroborate previous reports in high altitude (>4292 meters) railway workers, which have demonstrated increased susceptibility to AMS in those classified as overweight or obese based on BMI criteria $[2,8,9]$. The most likely explanation for these conflicting data is that the former cohort studies lacked a significant number of obese individuals to tease out a meaningful effect of body habitus on AMS development using a regression analysis. In a small group of young, yet inexperienced hikers, we found that the median BMI among those who reported severe AMS at 3800 meters was 27.5 vs. 22.3 among those who reported no or mild symptoms. Further, there was a significant correlation between BMI and self-reported AMS score at 4100 meters.

Using a hypobaric chamber to stimulate high-altitude exposure and hydrostatic weighing to quantify body fat, Ri-Li and colleagues [9] found that average AMS scores increased more with time spent in the chamber for obese men than for non-obese men. The pres- ent study extends this line of scientific inquiry by quantifying both total and regional body fat using state-of-the-art DXA imaging. Our findings of greater trunk, android and visceral fat mass among those with severe AMS compared to those with no/minimally symptomatic AMS (albeit not statistically significant) provide some insight into the relative importance of central adiposity in promoting high-altitude illness. It is generally understood that obesity is associated with sleep-disordered breathing [22] and nocturnal hypoxia and hypercapnia that may lead to AMS [23]. Indeed, lower $\mathrm{SaO}_{2}$ [9] and $\mathrm{PaO}_{2}$ [8] and higher $\mathrm{PaCO}_{2}$ [8] among obese individuals have been reported at altitude. Not surprisingly, we also found an inverse association between altitude and $\mathrm{SaO}_{2}$ across our sample. In this context, we emphasize that central adiposity may increase the severity of sleep-disordered breathing by elevating mechanical loads on the upper airway and blunting neuromuscular responses to upper airway obstruction [24]. Moreover, adipose tissue is an abundant source of pro-inflammatory cytokines, such as TNF- $\alpha$, and IL- 6 . These are markedly elevated in central obesity $[25,26]$, and their purported somnogenic activity [27] may lead to a depression in upper airway neuromuscular control. Interestingly, in our subsample of women who were fitted with a PPG-based device, none exhibited sleep-disordered breathing at sea level, yet all 3 women experienced an increased number of apneic events at altitude, consistent with the periodic breathing phenomenon typically observed upon acute ascent to high altitude [28]. It is difficult to determine whether periodic breathing is the predominant cause of AMS, especially in light of the findings by Nussbaumer-Ochsner and colleagues [29] who reported that the number of apnea/hypopneas experienced per night by young hikers upon arrival to 4556 meters increased with each successive night spent at this altitude, whereas AMS symptoms and self-reported sleep disturbances that were prominent on the first night improved with 3 days of acclimatization. Still, we find it intriguing that the woman in the present study with the greatest amount of visceral fat also exhibited the greatest number of apneic events and reported the highest Lake Louise score at 3800 meters. Although not definitive (please see Limitations), this finding provides proof of concept that the initial onset of symptoms of AMS may be more pronounced among those who distribute fat centrally perhaps due to the impact on sleep-disordered breathing. The strong associations we observed between higher Lake Louise scores at altitude and increased markers of central fat, but not total body fat percentage per se, lend further support to this theory. 


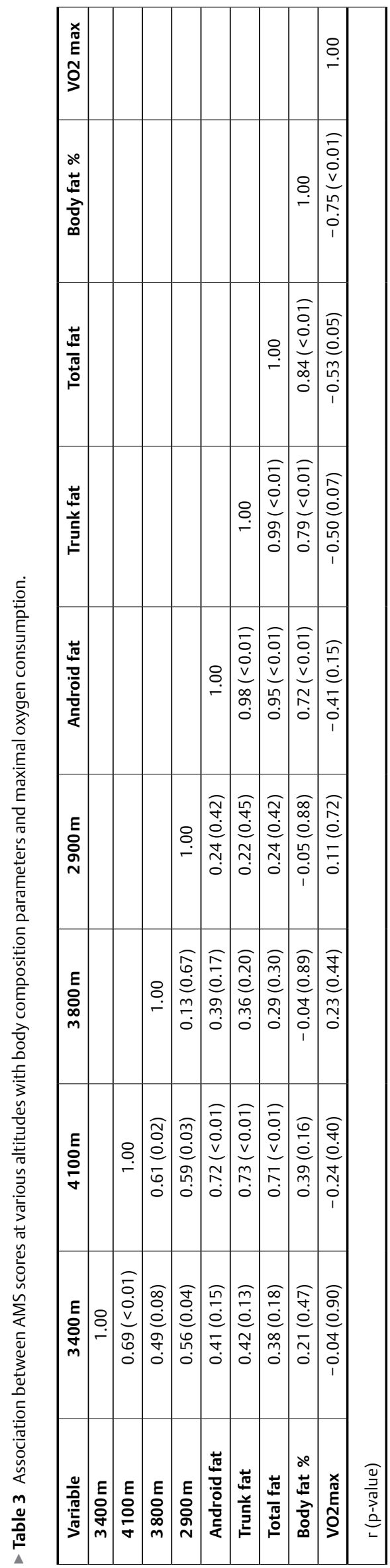

The above findings should be viewed in the context of several limitations. First, we did not measure blood markers in our sample, thereby precluding us from addressing a mechanistic link with empirical evidence between central adiposity and AMS. Second, the small sample size may have limited our power to detect significant differences in body fat parameters between those with no AMS vs. those with severe AMS. We therefore cannot discount the possibility that we made a type 2 error. Alternatively, given that only 2 subjects were overweight (BMI: 25.0-29.9) and one was obese $(\mathrm{BMI}: \geq 30)$ it is possible that this otherwise homogenous group could be contributing to the significant association we observed between AMS scores at altitude and body fat parameters. Yet, we feel these limitations are balanced by our sophisticated assessment of regional body fat with DXA imaging, thereby offering a clear advantage over previous studies that have relied on hydrostatic weighing or BMI. Third, we cannot be entirely sure that other environmental, motivational or physiological factors beyond the hypobaric environment may have worsened AMS symptoms reported by the participants. Of note, June is generally the coolest and driest time of year in this region of Peru. Indeed, the temperature on the first day of the trek was approximately $19{ }^{\circ} \mathrm{C}$. This dropped to near freezing (approximately $2{ }^{\circ} \mathrm{C}$ ) at night and increased to $24{ }^{\circ} \mathrm{C}$ the following day. Also, symptoms of AMS commonly intensify during periods of physical exertion because the metabolic requirements for oxygen increase substantially [30]. Aerobic capacity varied widely among members of the group and was inversely related to total body fat, but all participants ascended at the same rate (approximately 75 meters increase in altitude per hour). Thus, although we did not have reliable measures of exercise intensity, it can be assumed that those with lower fitness levels were hiking at a higher percentage of their maximal capacity. Also, some participants in the present study reported having chewed cocoa leaves during the trek, a natural remedy that is thought to alleviate altitude illness, although recent data would suggest otherwise [1]. Furthermore, since hiking guides provided meals, dietary content was similar across the sample (aside from the use of herbal supplements by some), yet the quantity of food and water ingested throughout the trek was difficult to quantify. Finally, although the Lake Louise Score is a well-validated tool to assess AMS [18], MacInnis and colleagues [31] found that the internal consistency of the score was improved by removing the sleep quality item, suggesting that impaired sleep should be considered separately from other AMS symptoms. All that being said, because this experiment was conducted in the field rather than an environmental chamber, we believe our findings are more generalizable to those persons partaking in recreational activities at high altitude.

In summary, an increased incidence of acute mountain sickness with increasing altitude was observed among young men and women making a high-altitude ascent. Those who experienced more severe AMS had more abdominal visceral, android and trunk fat, suggesting that central adiposity, rather than total body fat per se, is an important risk factor in the development of AMS. These findings suggest a need for obese individuals to take precautionary measures to reduce AMS risk. 


\section{Acknowledgements}

The authors thank the Towson University Study Abroad Office for logistical support.

\section{Conflict of Interest}

The authors declare that they have no conflict of interest.

\section{References}

[1] Salazar H, Swanson J, Mozo K, White AC Jr., Cabada MM. Acute mountain sickness impact among travelers to Cusco, Peru. J Travel Med 2012; 19: 220-225

[2] Wu TY, Ding SQ, Liu JL, Jia JH, Chai ZC, Dai RC. Who are more at risk for acute mountain sickness: a prospective study in Qinghai-Tibet railroad construction workers on Mt. Tanggula. Chin Med J (Engl) 2012; 125 : 1393-1400

[3] Schneider M, Bernasch D, Weymann J, Holle R, Bartsch P. Acute mountain sickness: influence of susceptibility, preexposure, and ascent rate. Med Sci Sports Exerc 2002; 34: 1886-1891

[4] Gaillard S, Dellasanta P, Loutan L, Kayser B. Awareness, prevalence, medication use, and risk factors of acute mountain sickness in tourists trekking around the Annapurnas in Nepal: a 12-year follow-up. High Alt Med Biol 2004; 5: 410-419

[5] Maggiorini M, Buhler B, Walter M, Oelz O. Prevalence of acute mountain sickness in the Swiss Alps. BMJ 1990; 301: 853-855

[6] Lupi-Herrera E, Seoane M, Sandoval J, Casanova JM, Bialostozky D. Behavior of the pulmonary circulation in the grossly obese patient. Pathogenesis of pulmonary arterial hypertension at an altitude of 2,240 meters. . Chest 1980; 78: 553-558

[7] Hirata K, Masuyama S, Saito A. Obesity as risk factor for acute mountain sickness. Lancet 1989; 2: 1040-1041

[8] Yang B, Sun Z], Cao F, Zhao H, Li CW, Zhang J. Obesity is a risk factor for acute mountain sickness: a prospective study in Tibet railway construction workers on Tibetan plateau. Eur Rev Med Pharmacol Sci 2015; 19: 119-122

[9] Ri-Li G, Chase PJ, Witkowski S, Wyrick BL, Stone JA, Levine BD, Babb TG. Obesity: associations with acute mountain sickness. Ann Intern Med 2003; 139: 253-257

[10] Wagner DR, Fargo JD, Parker D, Tatsugawa K, Young TA. Variables contributing to acute mountain sickness on the summit of Mt Whitney. Wilderness Environ Med 2006; 17: 221-228

[11] Ziaee V, Yunesian M, Ahmadinejad Z, Halabchi F, Kordi R, Alizadeh R, Afsharjoo HR. Acute mountain sickness in Iranian trekkers around Mount Damavand (5671m) in Iran. Wilderness Environ Med 2003; 14 : 214-219

[12] West JB, Peters RM Jr., Aksnes G, Maret KH, Milledge JS, Schoene RB. Nocturnal periodic breathing at altitudes of 6,300 and $8,050 \mathrm{~m}$. J Appl Physiol (1985) 1986; 61: 280-287
[13] Soriano-Co M, Vanhecke TE, Franklin BA, Sangal RB, Hakmeh B, McCullough PA. Increased central adiposity in morbidly obese patients with obstructive sleep apnoea. Intern Med J 2011; 41: 560-566

[14] Harriss DJ, Atkinson G. Ethical standards in sport and exercise science research: 2016 update. Int J Sports Med 2015; 36: 1121-1124

[15] Roach RC, Bartsch P, Hackett PH, Oelz O. The lake louise AMS scoring consensus committee. The Lake Louise acute mountain sickness scoring system. In: Sutton JR, Coates G, Houston CS. eds. Hypoxia and Molecular Medicine. Burlington: Queen City Printers; 1993: 272-274

[16] Kang J, Chaloupka EC, Mastrangelo MA, Biren GB, Robertson RJ. Physiological comparisons among three maximal treadmill exercise protocols in trained and untrained individuals. Eur J Appl Physiol 2001; 84: 291-295

[17] Tanaka H, Monahan KD, Seals DR. Age-predicted maximal heart rate revisited. J Am Coll Cardiol 2001; 37: 153-156

[18] Maggiorini M, Muller A, Hofstetter D, Bartsch P, Oelz O. Assessment of acute mountain sickness by different score protocols in the Swiss Alps. Aviat Space Environ Med 1998; 69: 1186-1192

[19] Romem A, Romem A, Koldobskiy D, Scharf SM. Diagnosis of obstructive sleep apnea using pulse oximeter derived photoplethysmographic signals. J Clin Sleep Med 2014; 10: 285-290

[20] Amir O, Barak-Shinar D, Henry A, Smart FW. Photoplethysmography as a single source for analysis of sleep-disordered breathing in patients with severe cardiovascular disease. J Sleep Res 2012; 21: 94-100

[21] Barak-Shinar D, Amos Y, Bogan RK. Sleep-disordered breathing analysis in a general population using standard pulse oximeter signals. Sleep Breath 2013; 17: 1109-1115

[22] Young T, Peppard PE, Taheri S. Excess weight and sleep-disordered breathing. J Appl Physiol 2005; 99: 1592-1599

[23] Becker HF, Piper AJ, Flynn WE, McNamara SG, Grunstein RR, Peter JH, Sullivan CE. Breathing during sleep in patients with nocturnal desaturation. Am J Respir Crit Care Med 1999; 159: 112-118

[24] Schwartz AR, Patil SP, Laffan AM, Polotsky V, Schneider H, Smith PL. Obesity and obstructive sleep apnea: pathogenic mechanisms and therapeutic approaches. Proc Am Thorac Soc 2008; 5: 185-192

[25] Arner P. Regional differences in protein production by human adipose tissue. Biochem Soc Trans 2001; 29: 72-75

[26] Dusserre E, Moulin P, Vidal H. Differences in mRNA expression of the proteins secreted by the adipocytes in human subcutaneous and visceral adipose tissues. Biochim Biophys Acta 2000; 1500: 88-96

[27] Opp MR. Cytokines and sleep. Sleep Med Rev 2005; 9: 355-364

[28] Weil JV. Sleep at high altitude. High Alt Med Biol 2004; 5: 180-189

[29] Nussbaumer-Ochsner Y, Ursprung ], Siebenmann C, Maggiorini M, Bloch KE. Effect of short-term acclimatization to high altitude on sleep and nocturnal breathing. Sleep 2012; 35: 419-423

[30] Frisancho AR. Developmental functional adaptation to high altitude: review. Am J Hum Biol 2013; 25: 151-168

[31] Macinnis M], Lanting SC, Rupert JL, Koehle MS. Is poor sleep quality at high altitude separate from acute mountain sickness? Factor structure and internal consistency of the Lake Louise Score Questionnaire. High Alt Med Biol 2013; 14: 334-337 